Arq. Bras. Med. Vet. Zootec., v.68, n.6, p.1705-1712, 2016

\title{
Glicerina bruta em dietas de juvenis de tambaqui
}

\author{
[Crude glycerin in diets of juvenile tambaqui] \\ P.R. Matos ${ }^{1}$, A.T. Ramos $^{2}$, S.E. Moron ${ }^{1 *}$ \\ ${ }^{1}$ Universidade Federal do Tocantins - (UFT) - Campus Araguaína - Araguaína, TO \\ ${ }^{2}$ Universidade Federal Santa Catarina - (UFSC) - Florianópolis, SC
}

\begin{abstract}
RESUMO
Este estudo verificou o nível de glicerina bruta em substituição total do óleo de soja e parcial do milho em dietas de juvenis de tambaqui sem que o ingrediente interfira no desempenho zootécnico desses animais. $\mathrm{O}$ trabalho foi realizado na Universidade Federal do Tocantins, avaliando cinco níveis de inclusão da glicerina bruta $(0 \% ; 7,5 \% ; 10 \% ; 12,5 \%$ e $15 \%)$ em três repetições com 10 animais cada ( $n=150$ tambaquis). Esses animais foram alimentados com as rações experimentais por um período de 75 dias (15 dias de adaptação e 60 dias experimentais). Ao final desse período, os animais foram contidos para a mensuração dos valores do desempenho zootécnico dos animais (taxa de crescimento específico, conversão alimentar aparente, eficiência alimentar, índice hepatossomático, ganho de peso e fator de condição). Durante o período experimental, não houve mortalidade, e de todos os índices avaliados apenas no fator de condição do animal ocorreu diferença estatística, quando se comparou o período inicial e o final do experimento. A inclusão de até $15 \%$ de glicerina bruta em dietas de tambaqui em substituição total do óleo de soja e parcial do milho pode ser realizada sem que prejudique o desempenho zootécnico da espécie estudada.
\end{abstract}

Palavras-chave: biodiesel, desempenho, eficiência alimentar, fator de condição, glicerol

\begin{abstract}
This study examined the level of crude glycerin in total replacement of soybean oil and partial replacement of corn in diets for tambaqui without ingredient interfering on the growth performance of these animals. The study was conducted at the Federal University of Tocantins, evaluating five levels of crude glycerin inclusion $(0 \%, 7.5 \%, 10 \%, 12.5 \%$, and $15 \%)$ in 3 replicates with 10 animals each ( $n=150$ tambaqui.) These animals were fed the experimental diets for a period of 75 days (15 days of adaptation and 60 days of trial), at the end of this period the animals were restrained to measure the values of the growth performance of animals (Specific growth rate, apparent food conversion, feed efficiency, hepatosomatic index, weight gain, and condition factor). During the study period, there were no deaths and in all indices evaluated, only the animal's condition factor presented statistical difference when comparing the initial and final periods of the experiment. The inclusion of up to $15 \%$ crude glycerin in tambaqui diets in total replacement of soybean oil and partial replacement of corn can be used without prejudice to the growth performance of the species studied.
\end{abstract}

Keywords: biodiesel, performance, feed efficiency, condition factor, glycerol

\section{INTRODUÇÃO}

O tambaqui, Colossoma macropomum, é uma espécie que possui excelente desempenho para cultivo em sistemas de criação intensiva, sendo mais cultivado na região Norte do Brasil (Melo et al., 2001). Além de apresentar bom crescimento, esse animal possui hábito gregário (Siqueira-Sousa et al., 2006) e é onívoro (Silva et al., 2007). Sua carne é saborosa e com boa

Recebido em 28 de maio de 2015

Aceito em 11 de março de 2016

*Autor para correspondência (corresponding author)

E-mail: sandromoron@uft.edu.br aceitação no mercado; quando estocada adequadamente, possui vida útil de 43 dias (Almeida et al., 2006). Quando criado em sistema intensivo, é alimentado com ração comercial, e, mesmo em cativeiro, esse animal apresenta boa conversão alimentar (Silva Júnior et al., 2011).

Para que os animais tenham um melhor aproveitamento do alimento que lhes é ofertado, este é formulado e balanceado com os mais 
diversos ingredientes e processos de elaboração (Silva et al., 2003). No intuito de reduzir o custo de produção, muitos alimentos alternativos são testados em rações experimentais, para que se possam incluir esses alimentos no banco de dados e formular rações comerciais de baixo custo (Rostagno et al., 2008). Para utilizar um alimento alternativo, deve-se levar em consideração sua disponibilidade, sua qualidade, os preços mais baixos do que os alimentos convencionais utilizados e o desempenho do animal quando alimentado desse modo (Bellaver, 2008).

Uma das maiores exigências na dieta para peixes é quanto ao fornecimento de energia (Graeff e Tomazelli, 2007). O glicerol foi indicado como fonte energética para suínos (Lammers et al., 2008a e aves (Lammers et al., 2008b); além disso, esse coproduto tem baixo valor econômico (Chi et al., 2007). Tal alimento possui alta absorção (Kerr e Dozier, 2008), devido ao fato de sua absorção se dar de forma passiva, o contrário de quando se utilizam outras fontes energéticas de ácidos graxos de cadeia longa que requerem a formação de micelas (Dozier et al., 2008).

O uso da glicerina na alimentação animal vem sendo cogitado em virtude da disponibilidade desse coproduto e da possibilidade de seu emprego na alimentação animal como ingrediente que corrobora bons índices de desempenho e baixo custo. Não consta na literatura o uso desse componente em peixes neotropicais, o que despertou o interesse na utilização desse produto na dieta de peixes nativos, como o tambaqui. Portanto, o presente trabalho verificou o nível de glicerina bruta na dieta de juvenis de tambaqui, sem que tal ingrediente interfira no desempenho zootécnico desses animais.

\section{MATERIAL E MÉTODOS}

O trabalho foi desenvolvido na Escola de Medicina Veterinária e Zootecnia da Universidade Federal do Tocantins - UFT, Campus de Araguaína - TO, conduzido de acordo com as normas éticas estabelecidas pela lei de Procedimentos para o Uso Científico de Animais e aprovado pela Comissão de Ética no Uso de Animais da UFT/Araguaína (protocolo 23101.002495/2014-82). Foram utilizados 150 juvenis de tambaqui, com peso inicial médio de $17,07 \pm 6,07 \mathrm{~g}$, distribuídos em 15 caixas de fibra com capacidade de 1000 litros (cinco tratamentos e três repetições, $n=10$ ), com aeração constante por meio de um compressor de ar e recirculação de água. $\mathrm{O}$ delineamento experimental utilizado foi em blocos ao acaso. No experimento, foram elaboradas cinco rações, sendo quatro níveis de inclusão $(7,5 \% ; 10 \% ; 12,5 \% ; 15 \%)$ de glicerina bruta em substituição ao óleo de soja e um tratamento controle como referência (sem inclusão de glicerina) (Tab. 1).

Tabela 1. Composição percentual e química das rações experimentais com diferentes níveis de glicerina bruta para juvenis de tambaqui

\begin{tabular}{|c|c|c|c|c|c|c|}
\hline \multirow[b]{2}{*}{ Ingredientes } & \multicolumn{6}{|c|}{ Níveis de inclusão } \\
\hline & 0 & & $7,5 \%$ & $10 \%$ & $12,5 \%$ & $15 \%$ \\
\hline Fubá de milho & 38,80 & & 31,80 & 29,30 & 26,8 & 24,3 \\
\hline Farelo de soja, $45 \%$ & 40 & & 40 & 40 & 40 & 40 \\
\hline Farinha de carne e ossos, $40 \%$ & 10 & & 10 & 10 & 10 & 10 \\
\hline Farelo de arroz & 10 & & 10 & 10 & 10 & 10 \\
\hline Óleo de soja & 0,5 & & 0 & 0 & 0 & 0 \\
\hline Glicerina bruta & 0 & & 7,5 & 10 & 12,5 & 15 \\
\hline Núcleo $^{1}$ & 0,75 & & 0,75 & 0,75 & 0,75 & 0,75 \\
\hline \multicolumn{7}{|c|}{ Atendimento } \\
\hline Proteína bruta $(\%)$ & & 26,46 & 26,43 & 26,47 & 26,44 & 26,45 \\
\hline Energia digestível (kcal/kg) & & 3127,20 & 3126,76 & 3127,65 & 3128,54 & 3129,43 \\
\hline Cálcio $(\%)$ & & 1,06 & 1,06 & 1,06 & 1,06 & 1,06 \\
\hline Fósforo (\%) & & 0,63 & 0,61 & 0,62 & 0,61 & 0,61 \\
\hline
\end{tabular}

${ }^{1}$ Níveis de micronutrientes por quilograma do produto: ácido fólico 20,25mg; antioxidante 66,15mg; cobalto $33,75 \mathrm{mg}$; cobre 337,50mg; ferro 337,50mg; iodo 50,62 mg; manganês $1350,00 \mathrm{mg}$; metionina 1,20mg; pantotenato de cálcio 315,56mg; selênio 10,12mg; sódio 55,58mg; tirosina 810,00mg; vit. A 216.000U.I; vit. B1 45.56mg; vit. B2 135,00mg; vit. B6 67,50mg; vit. C 600mg; vit. D3 50.625,00UI; vit. E 506.25UI; vit. H 2.70mg; vit. K3 50,62mg; vit. B12 675.00mcg; zinco $3375,00 \mathrm{mg}$. 
Os ingredientes foram moídos e peletizados em moedor de carne. Os peixes eram alimentados duas vezes por dia (oito horas e 17h), até a saciedade aparente, por um período de 75 dias (15 dias de adaptação e 60 dias de experimento).

Os parâmetros de qualidade de água, como $\mathrm{pH}$, oxigênio, temperatura, amônia e condutividade elétrica, foram aferidos semanalmente. Após o período experimental, os peixes foram mantidos em jejum por 24 horas para o esvaziamento do trato gastrointestinal para a realização das medidas individuais, com o intuito de se obterem os dados de desempenho zootécnico. Os animais foram insensibilizados por secção medular para posterior retirada de tecidos para as análises:

taxa de crescimento específico (TCE) - equivale ao crescimento diário dos animais, obtido em porcentagem. Para seu cálculo, utiliza-se a seguinte expressão (1):

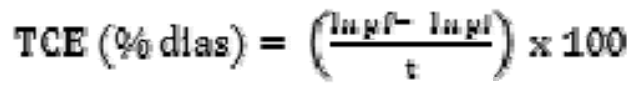

conversão alimentar aparente (CAA) - equivale à quantidade de ração necessária para o animal ganhar $1 \mathrm{~kg}$ de peso vivo (2):

$$
\mathrm{CAA}=\frac{\text { raçåo consumida }(\mathrm{g})}{\text { peso flnal }(\mathrm{g})-\text { peso } \ln \operatorname{lclal}(\mathrm{g})}
$$

eficiência alimentar (EA) - constitui o ganho de peso médio por peixe no grupo, dividido pelo consumo médio de ração por peixe. Trata-se da eficiência que o animal teve para converter a ração consumida em peso vivo (3):

$$
\mathrm{EA}=\frac{\text { ganho de massa }(\mathrm{g})}{\text { quantidade de raça } \operatorname{lngerdda(g)}} \times 100
$$

índice hepatossomático (IH) - é a relação entre o peso total do fígado e o peso total do peixe. Esse índice é obtido de acordo com a seguinte fórmula (4):

$$
I H=\frac{\text { peso do figado }(\mathrm{g})}{\text { peso corporal }(\mathrm{g})} \times 100
$$

ganho de peso (GP) - é o peso final do animal menos o peso inicial do animal. Esse cálculo é obtido pela seguinte fórmula (5):

\section{$\mathrm{GP}=$ pesofinal $(\mathrm{g})-$ peso inicial $(\mathrm{g})$}

fator de condição (FC) - mede indiretamente o estado fisiológico do animal em relação às reservas energéticas armazenadas, tais como glicogênio hepático e gordura corporal. Para sua determinação, usou-se a seguinte fórmula (6):

\section{$\mathrm{FC}=\frac{\text { massa }(\mathrm{g})}{\text { comprimento total }(\mathrm{cm})}$}

Os dados foram submetidos à análise de variância (ANOVA) e regressão, utilizando-se o programa Instat para Windows. Quando observadas diferenças significativas $(\mathrm{P}<0,05)$, foram aplicados o teste de média de Tukey, nos testes paramétricos, e nos não paramétricos, o teste de Kruskal-Wallis.

\section{RESULTADOS E DISCUSSÃO}

Não houve diferença nas variáveis ambientais quantificadas durante o experimento. Os valores médios para os parâmetros de qualidade da água registrados durante o período experimental estão expostos na Tab. 2.

Tabela 2. Parâmetros de qualidade da água durante o período experimental

\begin{tabular}{llllll}
\hline Parâmetros & Cont. & Trat. 1 & Trat. 2 & Trat. 3 & Trat. 4 \\
\hline pH & $6,7 \pm 0,43$ & $6,8 \pm 0,53$ & $6,5 \pm 0,25$ & $6,6 \pm 0,33$ & $6,4 \pm 0,73$ \\
Oxigênio dissolvido (mg/L) & $7,5 \pm 0,16$ & $7,1 \pm 0,93$ & $7,3 \pm 0,65$ & $7,6 \pm 0,44$ & $7,2 \pm 0,13$ \\
Temperatura $\left({ }^{\circ} \mathrm{C}\right)$ & $26,2 \pm 0,13$ & $26,74 \pm 0,06$ & $27,1 \pm 0,09$ & $25,56 \pm 0,2$ & $27,78 \pm 0,71$ \\
Amônia $(\mathrm{mg} / \mathrm{L})$ & $0,75 \pm 0,46$ & $0,92 \pm 0,78$ & $1,02 \pm 0,96$ & $0,98 \pm 0,59$ & $1,25 \pm 0,87$ \\
\hline
\end{tabular}

${ }^{1}$ Valores seguidos de médias e desvios-padrão.

Os valores obtidos de oxigênio dissolvido, temperatura, condutividade, $\mathrm{pH}$ e amônia mantiveram-se dentro dos padrões recomendáveis para o cultivo de peixes de clima tropical. 
Durante o período experimental, não ocorreu mortalidade em nenhum dos tratamentos estudados. Neu et al. (2012) constataram mortalidade de tilápias-do-nilo (Oreochromis niloticus) alimentadas com glicerol. Os maiores valores de mortalidade foram nos níveis de 7,5\%, seguidos pelo de $2,5 \%$, e o maior nível de sobrevivência foi no tratamento que não recebeu nenhuma adição de glicerol (grupo controle: cerca de $70 \%$ ).

$\mathrm{Na}$ comparação dos índices zootécnicos, observou-se que não houve diferença estatística em praticamente todos os índices. Os valores obtidos nas análises da taxa de crescimento específico, na conversão alimentar, na eficiência alimentar, no índice hepatossomático e no ganho de peso dos tambaquis não diferenciaram estatisticamente, indicando, assim, que todos obtiveram o mesmo ritmo de crescimento diário individual e de ganho de peso em grupo, com uma conversão alimentar semelhante durante todo o período experimental, e o índice hepatossomático demonstrou que os animais não necessitaram alterar as suas funções metabólicas do fígado para digerirem o ingrediente testado, mesmo sendo alimentados com diferentes tipos de rações (Tab. 3).

Tabela 3. Taxa de crescimento específico (TCE), conversão alimentar aparente (CAA), eficiência alimentar (EA), índice hepatossomático (IH) ganho de peso e fator de condição inicial (FCI) e final (FCF) de tambaquis alimentados com diferentes níveis de glicerina bruta

\begin{tabular}{|c|c|c|c|c|c|}
\hline $\begin{array}{l}\text { Glicerina } \\
\text { bruta (\%) }\end{array}$ & 0 & 7,5 & 10 & 12,5 & 5 \\
\hline TCE (\%) & $1,59 \pm 0,4 \mathrm{a}$ & $1,59 \pm 0,39 a$ & $1,52 \pm 0,36 a$ & $1,51 \pm 0,04 \mathrm{a}$ & $1,47 \pm 0,17 \mathrm{a}$ \\
\hline CAA & $1,02 \pm 0,06 \mathrm{a}$ & $1,27 \pm 0,15 \mathrm{a}$ & $1,29 \pm 0,18 \mathrm{a}$ & $1,22 \pm 0,3 \mathrm{a}$ & $1,53 \pm 1,06 \mathrm{a}$ \\
\hline EA $(\%)$ & $0,98 \pm 0,06 \mathrm{a}$ & $0,79 \pm 0,09 a$ & $0,78 \pm 0,1 \mathrm{a}$ & $0,85 \pm 0,18 \mathrm{a}$ & $0,87 \pm 0,49 a$ \\
\hline $\mathrm{IH}(\%)$ & $0,015 \pm 0,004 a$ & $0,019 \pm 0,003 \mathrm{a}$ & $0,017 \pm 0,003 a$ & $0,016 \pm 0,008 \mathrm{a}$ & $0,017 \pm 0,003 \mathrm{a}$ \\
\hline GP (\%) & $36,88 \pm 10,45 a$ & $38,15 \pm 14,92 \mathrm{a}$ & $34,09 \pm 11,58 \mathrm{a}$ & $33,43 \pm 11,39 a$ & $37,96 \pm 18,71 \mathrm{a}$ \\
\hline FCI (g/cm) & $1,97 \pm 0,14 \mathrm{aB}$ & $1,99 \pm 0,15 \mathrm{aB}$ & $1,97 \pm 0,29 \mathrm{aB}$ & $1,64 \pm 0,18 \mathrm{aB}$ & $2,19 \pm 0,25 \mathrm{aB}$ \\
\hline $\mathrm{FCF}(\mathrm{g} / \mathrm{cm})$ & $3,86 \pm 0,53 \mathrm{aA}$ & $4,26 \pm 0,9 \mathrm{aA}$ & $3,64 \pm 0,43 \mathrm{aA}$ & $3,9 \pm 0,51 \mathrm{aA}$ & $4,63 \pm 0,84 \mathrm{aA}$ \\
\hline
\end{tabular}

*Letras minúsculas iguais não diferem na mesma linha.

**Letras maiúsculas diferentes diferem na mesma coluna.

Neu et al. (2012), ao testarem o desempenho zootécnico de tilápias-do-nilo alimentadas com glicerol, verificaram que também não houve diferença significativa na taxa de crescimento específico, na conversão alimentar, no ganho de peso, no comprimento final e no peso final dos animais. Porém, a conversão alimentar dos animais estudados teve uma conversão alimentar consideravelmente alta, um possível resultado das baixas temperaturas durante o período experimental.

Ao trabalharem glicerina bruta em outras espécies de monogástricos, como frangos da linhagem Cobb, Guerra et al. (2011) verificaram um efeito quadrático, sendo o decréscimo ao nível de 5\% no peso médio aos 42 dias e na conversão alimentar à medida que foram aumentados linearmente os níveis de 0 a $10 \%$ de inclusão de glicerina bruta na dieta. Assim, constataram que a glicerina bruta para frangos da linhagem Cobb pode ser incluída na dieta em valores de até $5 \%$.
Batista (2010), quando testou glicerol vegetal bruto e glicerol vegetal semipurificado para codornas de um a 14 dias e de 15 a 35 dias, não observou diferença no consumo de ração e no ganho de peso em ambos os tipos de glicerol. Entretanto, a conversão alimentar das aves aumentou linearmente com o aumento dos níveis de 0 a $16 \%$, considerando que, dependendo da viabilidade econômica de ambos os gliceróis testados, podem ser incluídos até $16 \%$ deles em dietas de codornas tanto de 1 a 14 dias, como de 15 a 35 dias.

Piano (2012), ao testar a inclusão de até $12 \%$ de glicerina semipurificada mista na dieta de leitões na fase inicial (15 a $30 \mathrm{~kg}$ ), observou que tal inclusão não prejudicou o desempenho zootécnico desses animais. Resultado semelhante foi encontrado por Groesbeck et al. (2008), ao incluírem até $12 \%$ de glicerina bruta em dietas para leitões, verificaram que não houve diferença no índice de conversão alimentar e observarem que os índices de ganho de peso diário e o consumo diário de ração aumentaram 
linearmente em consideração aos outros níveis de inclusão. Gallego (2012), quando testou glicerina semipurificada neutralizada para leitões na fase inicial (15-30kg) e nas fases de crescimento e de terminação $(30-90 \mathrm{~kg})$, sugeriu a utilização de até $14 \%$ desse coproduto na ração, uma vez que esse nível não afetou o desempenho e as variáveis plasmáticas do animal.

O uso de glicerol na alimentação animal também foi estudado nas espécies ruminantes. A suplementação com glicerina bruta a $10 \mathrm{~g} / \mathrm{kg}$ de peso vivo animal teve bons resultados na ingestão de matéria seca e no ganho de peso de bezerros Holandeses com idade média de 6,6 meses alimentados com silagem de sorgo. Chilibroste et al. (2011) encontraram ganhos similares na suplementação com milho $(0,518 \mathrm{~kg} / \mathrm{dia})$ e glicerina bruta $(0,571 \mathrm{~kg} / \mathrm{dia})$ e concluíram que a suplementação pode ser feita tanto com milho quanto com glicerina.

Napoles (2012), quando testou níveis de 0, 5 e $10 \%$ de glicerina bruta em substituição ao milho em dietas de bezerros machos da raça Holandês, não verificou diferença nas variáveis de consumo e ganho de peso diário. Em estudo com bezerros recém- desaleitados de corte, Gunn et al. (2011) testaram os níveis de 0,15 e $30 \%$ de glicerina bruta combinados com grãos secos solúveis de destilaria e observaram que os níveis reduziram linearmente o consumo de matéria seca, porém os autores sugeriram que a substituição do milho pela glicerina pode ser de até $15 \%$.

Lage et al. (2010) concluíram em seu trabalho que a inclusão de até $6 \%$ de glicerina bruta na dieta de cordeiros em terminação melhorou a conversão alimentar e reduziu o custo com o ganho de carcaça desses animais.

A glicerina é um coproduto com alto valor energético e, quando testada, é substituída pelos carboidratos e lipídios das rações. Almeida (2010), ao trabalhar com diferentes níveis de carboidrato e lipídio na dieta de tambaqui, verificou que a dieta I $(30,5 \%$ de carboidrato e
$13,7 \%$ de lipídio) foi a que apresentou a melhor taxa de crescimento específico para o tambaqui nas condições de criação do estudo. Em estudos feitos com alevinos de tambaqui, em que foram utilizados diferentes níveis de energia e foi usado como fonte energética o óleo de soja, notou-se um ganho médio diário entre 1,32 e 1,61g/dia, sendo superior ao grupo controle (Camargo et al., 1998).

Pereira et al. (2011), ao testarem a interação de diferentes fontes e níveis de lipídios (óleo de soja e de dendê) na alimentação do híbrido tambacu (macho Piaractus mesopotamicus $\mathrm{x}$ fêmea Colossoma macropomum), verificaram que não houve diferença quanto às variáveis de desempenho zootécnico, peso final, ganho de peso diário, taxa de crescimento e sobrevivência. Entretanto, foi observado que houve um comportamento linear crescente nas variáveis de consumo diário de ração, consumo diário de proteína e conversão alimentar aparente.

Losenkann et al. (2008) não verificaram diferença estatística nas taxas de crescimento e de sobrevivência para jundiás submetidos aos níveis de 5 e $10 \%$ de fontes de óleos de canola, arroz ou soja. Melo et al. (2001), ao testarem diferentes fontes de lipídios (óleo de canola, fígado de bacalhau e banha suína) na alimentação de alevinos de jundiá (Rhamdia quelen), demonstraram que não houve diferença estatística nos valores de desempenho dos animais alimentados com essas diferentes fontes de lipídios. Entretanto, nos animais alimentados com $10 \%$ de óleo de canola, foi observada uma tendência de maior média de peso.

O fator de condição do animal foi o único índice de desempenho zootécnico que apresentou diferença significativa entre o período inicial e o período final do experimento, demonstrando, assim, que o animal modificou as suas reservas de glicogênio hepático e de gordura corporal durante o período experimental. Porém, tal fator não se diferenciou entre os tratamentos quando comparados nos mesmos períodos (Fig. 1). 


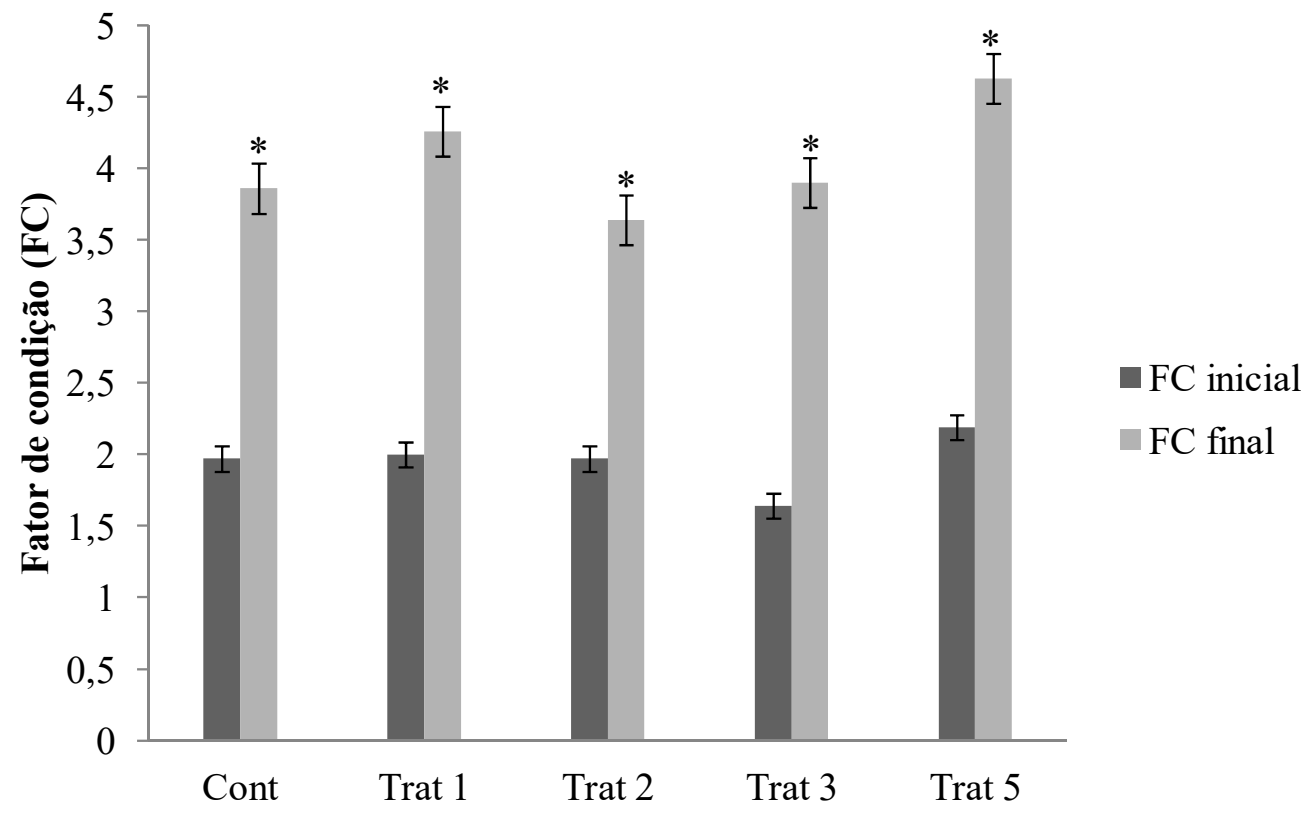

Figura 1. Fator de condição inicial e final dos juvenis de tambaqui alimentados com doses crescentes de glicerina bruta $(0 \% ; 7,5 \% ; 10 \% ; 12,5 \%$ e $15 \%$, respectivamente).

Melo et al. (2001) observaram que o fator de condição de alevinos de jundiá não foi alterado quando estes foram submetidos a dietas com diferentes níveis e fontes de lipídios. Losekann et al. (2008), ao estudarem juvenis de jundiá alimentados com diferentes fontes e níveis de óleos vegetais, verificaram que a deposição de gordura foi maior $(25,65 \%)$ nos animais alimentados com óleo de soja comparados com os animais alimentados com óleo de arroz e superior $(133,06 \%)$ quando comparados com os animais alimentados com óleo de canola, tornando, assim, o fator de condição maior nos animais alimentados com óleo de soja. Também ao estudarem a mesma espécie, porém em diferente fase (alevinos), Lopes et al. (2006) observaram um efeito linear da variável fator de condição, bem como o fato de que quanto maior o nível de energia da dieta maior o fator de condição dos animais.

\section{CONCLUSÃO}

A inclusão de até $15 \%$ de glicerina bruta em dietas de tambaqui em substituição total do óleo de soja e parcial do milho pode ser utilizada sem que possa prejudicar o desempenho zootécnico da espécie estudada, pois os valores desses índices não se diferenciaram do tratamento com nível $0 \%$ de inclusão.

$\mathrm{O}$ único índice que se diferenciou durante o período experimental foi o fator de condição, porém essa diferença ocorreu durante o período (inicial e final) do experimento e não entre os tratamentos estudados, o que sugere que o animal teve um maior armazenamento de suas reservas energéticas.

\section{REFERÊNCIAS}

ALMEIDA, L.C. Desempenho produtivo, eficiência digestiva e perfil metabólico de juvenis de tambaqui, Colossoma macropomum (Cuvier, 1808) alimentados com diferentes taxas carboidrato/lipídio. 2010. 103f. Tese (Doutorado) - Universidade Federal de São Carlos, São Carlos, SP.

ALMEIDA, N.M.; BATISTA, G.M.; KODAIRA, M.; LESSI, E. Alterações postmortem em tambaqui (Colossoma macropomum) conservados em gelo. Ciênc. Rural, v.36, p.12881293, 2006. 
BATISTA, E. Avaliação nutricional do glicerol para codornas de corte. 2010. 69f. Dissertação (Mestrado em Zootecnia) - Centro de Ciências Agrárias, Universidade Estadual de Maringá, Maringá, PR.

BELLAVER, C. Sistemas de produção de frangos de corte: nutrição e alimentação. Santa Catarina: Embrapa Suínos e Aves, 2008. Disponível em: $<$ http://sistemasdeprodução.cnptia.embrapa.br/Fo ntesHTML/Ave/ProducaodeFrangodeCort//Alim entos.html>. Acessado em: 20 abril 2013.

CAMARGO, A.C.S.; VIDAL JUNIOR, M.V.; DONZELE, J.L. et al. Níveis de energia metabolizável para Tambaqui Colossoma macropomum (CUVIER, 1818) dos 30 aos 180 gramas de peso vivo. Rev. Bras. Zootec., v.27, p.409-415, 1998.

CHI, Z.; PYLE, D.; WEN, Z. et al. A laboratory study of producing docosahexaenoic acid from biodiesel-waster glycerol by microalgal fermentation. Process Biocherm., v.42, p.15371543, 2007.

CHILIBROSTER, P.; ELIAS, A.; MARCHELLI, J.P. Use of com or crude glycerol as energy source to supplement Holstein calves fed with sorghum silage ad-libitum. J. Anim. Sci., v.89, p.198, 2011.

DOZIER, W.A.; KERR, B.J.; CORZO, A. et al. Apparent metabolizable energy of glycerin for broiler chickens. J. Poult. Sci., v.87, p.317-322, 2008.

GALLEGO, A.G. Glicerina semipurificado neutralizada na alimentação de suínos (2012). 2012. 77f. Dissertação (Mestrado em Zootecnia) - Centro de Ciências Agrárias, Universidade Estadual de Maringá, Maringá. SC.

GRAEFF, A.; TOMAZELLI, A. Fontes e níveis de óleo na alimentação de carpa comum (Cyprinus carpio L.) na fase de crescimento. Ciênc. Agrotec., v.31, p.1545-1551, 2007.

GROESBECK, C.N.; MCKINNEY, L.J.; DEROUCHEY, J. et al. Effect of crude glycerol on pellet mill production and nursery pig growth performance. J. Anim. Sci. v.86, p.2228-2236, 2008.
GUERRA, R.L.H.; MURAKAMI, A.E.; GARCIA, A.F.Q.M. et al. Glicerina bruta mista na alimentação de frangos de corte (1 a 42 dias). Rev. Bras. Saúde Prod. Anim., v.12, p.10381050, 2011.

GUNN, P.J.; LEMANAGER, R.P.; BUCKMASTER, D.R. et al. Effects of dried distiller's grains with solubles on performance, carcass characteristics, and metabolic parameters of early weaned beef calves. Prof. Anim. Sci., v.27, p.283-294, 2011.

KERR, B.J.; DOZIER, W.A. Crude glycerin for monogastrics feeds. Rend. Mag., v.37, p.10-11, 2008.

LAGE, J.F.; PAULINO, P.V.R.; PEREIRA, L.G.R. et al. Glicerina bruta na dieta de cordeiros terminados em confinamento. Pesqui. Agropecu. Bras., v.45, p.1012-1020, 2010.

LAMMERS, P.J.; KERR, B.J.; WEBER, T.E. et al. Growth performance, carcass characteristics, meat quality, and tissue histology of growing pigs fed crude glycerin supplemented diets. $J$. Anim. Sci., v.86, p.2962-2970, 2008a.

LAMMERS, P.J.; KERR, B.J.; HONEYRNAN, M.S. et al. Nitrogen corrected apparent metabolizable energy value of crude glycerol for laying hens. J. Poult. Sci., v.87, p.104-107, 2008 b.

LOPES, P.R.S.; POUEY, J.L.O.F.; ENKE, D.B.S. et al. Desempenho de alevinos de jundiá Rhamdia quelem alimentados com diferentes níveis de energia na dieta. Biodivers. Pampeana, v.4, p.32-37, 2006.

LOSEKANN, M.E.; RANDUNZ NETO, J.; EMANUELLI, T. et al. Alimentação do jundiá com dietas contendo óleos de arroz, canola ou soja. Ciênc. Rural, v.38, p.225-230, 2008.

MELO, J.F.B.; RADUNZ NETO, J.; SILVA, J.H.S. Uso de diferentes fontes e níveis de lipídios na alimentação de alevinos de jundiá (Rhamdia quelen). Pesqui. Agropecu. Gaúcha, v.7, p.127-134, 2001.

MELO, L.A.S.; IZEL, A.C.U.; RODRIGUES, F.M. Criação de tambaqui (Colossoma macropomum) em viveiros de argila/barragens no estado do Amazonas. Manaus: Embrapa Amazônia Ocidental, 2001. 25p. (Documentos Embrapa Amazônia Ocidental, n.18). 
NAPOLES, G.G.O. Desempenho e metabolismo de bezerros leiteiros em aleitamento convencional recebendo concentrado inicial contendo glicerol em substituição ao milho. 2012. 92f. Dissertação (Mestrado) - Escola Superior de Agricultura Luiz de Queiroz, Universidade de São Paulo, Piracicaba, SP.

NEU, D.H.; FURUYA, W.M.; YAMASHIRO, D. et al. Glicerol na dieta de alevinos de tilápias do Nilo. Rev. Agrar., v.5, p.288-294, 2012.

PEREIRA, M.C.; AZEVEDO, R.V.; BRAGA, L.G.T. Óleos vegetais em rações para híbrido Tambacu (macho Piaractus mesopotamicus $\mathrm{x}$ fêmea Colossoma macropomum). Rev. Bras. Saúde Prod. Anim., v.12, p.551-562, 2011.

PIANO, L.M. Glicerina semipurificada na alimentação de suínos. 2012. 90f. Tese (Doutorado em Zootecnia) - Centro de Ciências Agrarias, Universidade Estadual de Maringá, Maringá. PR.

ROSTAGNO, H.S.; NASCIMENTO, A.H.; ALBINO, L.F.T. et al. Retrospectiva e desafios da produção animal. aves e suínos. Viçosa: DZO/U.F.V, 2008. Disponível em: $<$ www.sbz.org.br/eventos/PortoAlegre/homepag esbz/Horacio.htm>. Acessado em: 20 abril 2013.
SILVA JUNIOR, W.A.; SILVA, C.N.; PENAFORT, J.M. et al. Alevinos de tambaqui (Colossoma macropomum) alimentados com ração comercial incrementada por abóbora (Cucúrbita moschata). In: SEMINÁRIO ANUAL DE INICIAÇÃO CIENTÍFICA, 9., 2011, Parauapebas. Anais... Parauapebas: UFRA, 2011.

SILVA, J.A.M.; PEREIRA FILHO, M.; CAVERO, B.A.S. Digestibilidade aparente dos nutrientes e energia de ração suplementada com enzimas digestivas exógenadas para juvenis de tambaqui (Colossoma macropomum Cuvier, 1818). Acta Amazonica, vol.37, p.157-164, 2007.

SILVA, J.A.M.; PEREIRA FILHO, M.; OLIVEIRA, M.I.P. Frutos e sementes consumidos pelo tambaqui, Colossoma macrompum (Cuvier, 1818) incorporados em rações. Digestibilidade e velocidade de trânsito pelo trato gastrointestinal. Rev. Bras. Zootec., v.32, p.1815-1824, 2003.

SIQUEIRA, F.K.S; BARBOSA, R.P.; FREITAS, C.E.C. Peixes do médio rio Negro. Manaus: EDUA, 2006. 43p. 